\title{
Der Tod ist kein Zufall: Ungleiche Lebenszeitchancen als Herausforderung für die Sozialpolitik
}

\author{
Michael NOLLERT ${ }^{1}$ \\ Universität Fribourg
}

\begin{abstract}
Menschen sind vor dem Tod ungleich, und zwar nicht nur hinsichtlich sozialem Status, sondern auch im Hinblick auf ihre Lebenszeitchancen. Menschen mit geringen Lebenschancen verfügen über weniger Bildung, Einkommen, Vermögen und sterben früher als Menschen mit grösserer Ressourcenausstattung. Dieser Zusammenhang lässt sich sowohl global als auch innerhalb von reichen Ländern wie etwa der Schweiz nachweisen. Der vorliegende Beitrag gibt zunächst einen Überblick über die Ursachen ungleicher Lebenszeitchancen. Danach folgt ein Überblick über ausgewählte empirische Analysen. Abschliessend wird auf sozialpolitische Aspekte fokussiert. So liegt es auf der Hand, dass ein fixes Rentenalter, sei das 65 oder 67 Jahre, vor allem jene Menschen benachteiligt, die über ein hohes Risiko verfügen, unterdurchschnittlich früh zu sterben.
\end{abstract}

Keywords: Lebenszeitchancen, Mortalität, soziale Ungleichheiten, Rentenalter

\section{Der König:}

Höhnst du also meine Hoheit,

Daß du's wagst, voran zu gehen?

Hast du gar so schnell verloren

All' Erinnern, plumper Bettler,

Daß du als mein Knecht geboren?

Der Bettler:

Deine Rolle ist zu Ende

In des Grabes Garderobe

Sind wir all' einander gleich;

Was du warst, kann wenig frommen.

(Calderón de la Barca, 1665, 1924 übersetzt von Eichendorff und Lorinser)

1 Michael Nollert, Prof. Dr., Universität Fribourg, michael.nollert@unifr.ch

Studium der Soziologie, Politikwissenschaft und Publizistikwissenschaft an der Uni Zürich, Promotion und Habilitation an der Universität Zürich, seit 2001 Professor an der Universität Fribourg und seit 2002 Privatdozent am Soziologischen Institut der Universität Zürich. Forschungsschwerpunkte: soziale Netzwerke, soziale Ungleichheiten, Sozialpolitik, Wirtschaftssoziologie, Arbeitsmarkt, soziale Konflikte 


\section{Einleitung}

Dass alle Menschen irgendwann sterben, mag Bettler über die Tatsache hinweg trösten, dass Menschen vor dem Tod ungleich sind und Bettler zudem in der Regel früher sterben. Entsprechend haben in traditionalen Gesellschaften allen voran Vertreter religiöser Institutionen versucht, die weniger privilegierten Schichten zu beruhigen und von einer Reform oder gar Revolution der Sozialstruktur abzuhalten. So suggeriert auch Calderón de la Barcas Welttheater (1655), dass uns Gott alle, ob arm oder reich, auffordert, unsere kurze Lebenszeit im Sinne der Bibel zu nutzen. Denn: vor Gottes Gericht seien wir letztlich alle gleich, zumal auch ein Kamel eher durch ein Nadelöhr als ein Reicher ins Himmelreich gelangt. Kurzum: Ein reiches, langes Leben schütze also nicht a priori vor dem Fegefeuer.

In der Tat waren traditionale Gesellschaften nicht bloss durch soziökonomische Ungleichheiten, sondern auch durch extreme Lebenszeitungleichheiten geprägt. Perrenoud (1975) zufolge erreichten im 17. Jahrhundert etwa im urbanen Kontext Genf von 1000 Angehörigen der Oberschicht (höhere Amtsträger, Gross- und mittleres Bürgertum) immerhin 305 das 60. Lebensjahr. In der Mittelschicht (Kleinbürgertum, Handwerker, qualifizierte Arbeiter) waren es 171 und in der Unterschicht (unqualifizierte Arbeiter, Handlanger) waren es gar nur 106.

Erst mit dem Fortschritt der Medizin und sozialpolitischen Reformen im späten 19. Jahrhundert beginnt sich der Zusammenhang zwischen sozialem Status und Mortalität allmählich zu verringern. Verantwortlich dafür war vorab die Schaffung moderner Gesundheitssysteme, die nicht nur zur Anhebung der durchschnittlichen Lebenserwartung, sondern auch zur Verringerung der medizinischen Versorgungsunterschiede beitrugen.

Unberücksichtigt blieb bei der Konstruktion der universellen Altersvorsorge indes das Faktum, dass auch in den wohlhabenden OECD-Staaten nach wie vor Arme und weniger Gebildete früher sterben und folglich auch weniger lang Rente beziehen.

Obwohl sich die statusbedingten Lebenszeitchancen im 20. Jahrhundert dank des modernen Wohlfahrtsstaats markant verringert haben, ist absehbar, dass eine neoliberale Gesundheitspolitik, die die gesundheitlichen Ungleichheiten zwischen den sozialen Schichten akzentuiert, diesen Trend bremsen wird (siehe Therborn 2013). Einige Studien sprechen gar dafür, dass die Lebenserwartung im 21. Jahrhundert wieder verstärkt mit dem sozialen Status zusammenhängt.

Der vorliegende Beitrag hat drei Teile. Vorab werden empirische Befunde präsentiert, wobei die Situation in der Schweiz im Vordergrund steht. Der zweite Teil verweist auf die Relevanz des Themas im Kontext der schweizerischen Gesundheitspolitik und fokussiert auf die Gründe dafür, weshalb sich soziale Statusunterschiede in unterschiedlichen Lebenszeiten niederschlagen. Im dritten Teil wird die sozialpolitische Relevanz der sozial bedingten Mortalitätsunterschiede thematisiert. Dabei wird betont, dass in der Debatte über die Altersvorsorge das Faktum ignoriert wird, dass strukturelle benachteiligte Menschen früher sterben und folglich auch weniger lang Rente beziehen (vgl. Künzler/Knöpfel 2002; Wanner 2012).

Der vorliegende Beitrag zielt vor allem auch darauf ab, zwei Themen in den Vordergrund zu rücken, die in der sozialarbeiterischen und sozialpolitischen Debatte vernachlässigt werden. Zum einen fokussieren wir im Unterschied zum Sterbehilfediskurs, der sich auf die ungewollte Verlängerung der Lebenszeit konzentriert, auf die ungewollte Verkürzung der Le- 
benszeit infolge struktureller Benachteiligung. Zum andern betonen wir im Unterschied zum hegemonialen Diskurs über die finanziellen Probleme der Altersvorsorge, dass die Lebenszeitchancen und damit auch die Chancen, möglichst lang Rente zu beziehen, ungleich verteilt sind.

\section{Sozialer Status und Lebenszeitdauer}

Die Forschung zum Zusammenhang zwischen sozialem Status und Lebenszeitdauer lässt sich in zwei Hauptbereiche gliedern. Individualdatenorientierte Analysen fokussieren auf Merkmale von Menschen, und zwar analog zu den geläufigen Statuszuweisungsmodellen sowohl askriptive als auch erworbene. Eine weitere Gruppe von Analysen thematisiert Faktoren, die die Lebenszeitunterschiede in einem Kontext, sei das eine Gemeinde oder ein Land, beeinflussen. Entsprechend stehen hier Kontextfaktoren wie das BIP pro Kopf oder die ökonomische Ungleichheit im Vordergrund.

In der vergleichenden Forschung, aber auch von Lebens- und Sozialversicherungen werden je nach Fragestellung in der Regel zwei Indikatoren berücksichtigt: Die Sterblichkeit (Mortalität) wird anhand der Häufigkeit von Todesfällen oder Gestorbenen in einer spezifischen Population berechnet. So kalkuliert etwa das Bundesamt für Statistik (BFS) seit 1876/1880 nach jeder Eidgenössischen Volkszählung die so genannten grossen oder allgemeinen Sterbetafeln für die Wohnbevölkerung der Schweiz. Vor allem im Diskurs über die Altersvorsorge und in den Weltentwicklungsberichten dominiert dagegen die Lebenserwartung. Damit gemeint ist die durchschnittliche Zahl der momentan zu erwartenden (weiteren) Lebensjahre eines Menschen unter der Voraussetzung, dass die gegenwärtigen altersspezifischen Sterbeziffern konstant bleiben.

Ein zentrales askriptives Merkmal, das in den meisten empirischen Analysen signifikant mit der Mortalität und Lebenserwartung korreliert, ist das Geschlecht. So leben Frauen in allen sozialen Schichten länger als Männer. Lebensverlängernd wirken allen voran auch eine hohe Bildung und ein hohes Einkommen (z.B. Voges/Groh-Samberg 2011; Lampert/Kroll 2014; Sasson 2015; Chetty et al. 2016) und in den USA selbstverständlich eine weisse Hautfarbe. In der vergleichenden Forschung kaum beachtet sind bislang die Auswirkungen des sozialen Kapitals. Aus der Gesundheitsforschung wissen wir immerhin, dass gute starke Bindungen (strong ties: FreundInnen und Familie) den Verlauf von Krankheiten positiv beeinflussen (vgl. Poder/He 2010; Eriksson 2011; Rocco/Suhrcke 2012; Hartung 2014).

Geht man davon aus, dass ein Grossteil des ökonomischen, kulturellen, aber auch des sozialen Kapitals vererbt wird, liegt es auf der Hand, dass die Lebenszeitchancen vom sozialen Herkunftsmilieu bestimmt werden. Besonders deutlich erkennbar werden diese Chancenungleichheiten bei den Zusammenhängen zwischen der sozialen Lage der Mütter und der Säuglings- und Kindersterblichkeit (z.B. Human Development Report 2005).

Das Ausmass der schichtspezifischen Säuglingssterblichkeit ist zweifellos ein valider Indikator dafür, inwiefern in einem Kontext die medizinische Versorgung auch für einkommensschwache Menschen zugänglich ist. Über die ungleiche Zugänglichkeit informieren auch Vergleiche des Zusammenhangs zwischen der sozialstrukturellen Position und der Mortalität. So spricht etwa die Pionierstudie von Mackenbach et al. (2008) dafür, dass der Zusammenhang, 
gemessen an prozentualen Unterschieden in der Mortalität zwischen sozialen Schichten, in den osteuropäischen Ländern vergleichsweise eng, in Spanien, aber auch in der Schweiz dagegen etwas geringer ist.

In einer neueren Studie berücksichtigten Mackenbach et al. (2016) auf der Basis von Daten zu elf Ländern zwei unterschiedliche Ungleichheitsindizes und deren Trends zwischen 1990 und 2010: die Unterschiede in der Mortalität zwischen den Schichten einerseits in Prozenten und andererseits in absoluten Jahren. Dabei zeigt sich, dass sich mit Ausnahme der untersten Schicht in Litauen in allen Ländern bei allen Schichten sowohl eine relative als auch eine absolute Verringerung der Mortalität beobachten lässt. Allerdings variieren die Trends je nach Ungleichheitsindex. Während bei beiden Geschlechtern die prozentuale Verringerung der Mortalität bei der höchsten Schicht grösser war als bei der tiefsten, ist bei der Verringerung der Mortalität gemessen an den erreichten Lebensjahren kein einheitliches Bild erkennbar. So gewinnen sowohl Männer als auch Frauen mit tiefem Status in den meisten Ländern mehr Lebensjahre hinzu als Männer und Frauen mit hohem Status. Das trifft jedoch nicht auf Finnland (beide Geschlechter) sowie Schweden, Norwegen, Frankreich, Schweiz und Slowenien (bezüglich Frauen) zu.

Dass in den letzten Jahren die Unterschiede zwischen den Ländern mit Ausnahme der in Lebensjahren gemessenen Mortalität allerdings eher grösser als kleiner geworden sind, belegen vor allem Studien aus den Deutschland und den USA, zwei Länder, die von Mackenbach et al. nicht berücksichtigt wurden. So zeigen z.B. Lampert und Kroll (2014), dass die Lebenserwartung der 65-Jährigen zwischen 1995 und 2008 bei der höchsten Einkommensschicht um 2.8 Jahre, bei der tiefsten Einkommensschicht dagegen nur um 1.1 Jahre gestiegen ist. In den neuen Bundesländern beträgt dieses Gefälle gar $3.8 \mathrm{zu} 1.7$ Jahre. Eindeutig sind auch die Trends bezüglich des Effekts der Bildung in den USA. So belegt Sasson (2016), dass die Lebenserwartungskluft zwischen tiefen und hohen Bildungsschichten zwischen 1990 und 2010 markant angestiegen ist.

Im Unterschied zur Gesundheit sind schichtspezifische Mortalitäten und Lebenserwartungen in der Schweiz noch nicht breit erforscht. Spätestens seit der Pionierstudie von Guberan und Usel (2000) auf der Basis von Daten zum Kanton Genf wissen wir immerhin, dass das Mortalitäts- und Behinderungsrisiko zwischen sozioprofessionellen Klassen und Berufsgruppen auch hierzulande stark variiert. So zeigte sich u.a., dass die Mortalität von Männern zwischen 45 und 65 Jahren bei Angelernten und Hilfsarbeitskräften $20.5 \%$, bei den freien und wissenschaftlichen Berufen dagegen nur $13.2 \%$ betrug. Analog variiert die Lebenserwartung beider Gruppen: 66.2 Jahre versus 70.6 Jahre. Bei den Berufen verfügten die Artisten, die Fabrikarbeiter und besonders die RaumpflegerInnen, Hauswarte, Streckenarbeiter über hohe Mortalitätsrisiken. Gering war das Risiko, zwischen 45 und 65 Jahren zu sterben, hingegen bei den Landwirten, Wissenschaftern und Lehrern.

Hohe Mortalitätsrisiken waren gemäss Wanner (2012) 2000-2005 bei den Männern vor allem im Gastgewerbe, in der Metallbranche und bei Maschinisten zu beobachten, wobei die Männer in einigen Berufen doppelt so hohe Sterblichkeiten erreichten wie die Frauen. Bei den Frauen waren vornehmlich Beschäftigte im Gastgewerbe und im Transportwesen gefährdet. Von Todesfällen gleichermassen verschont sind bei den Männern und Frauen vor allem LehrerInnen und ProfessorInnen. 
Differenziert man nach sozioprofessionellen Kategorien zeigt sich eine hohe Gefährdung von Frauen im obersten Management, bei den Selbständigen und den Arbeitern und Hilfsarbeitskräften, bei den Männern bei den Hilfsarbeitskräften, bei den Arbeitern und bei den Angestellten. Eine tiefe Mortalität finden wir dagegen bei den weiblichen und männlichen Ingenieuren und technischem Kader sowie bei freien Berufen. Zudem ist erkennbar, dass bei den Hilfsarbeitskräften, Arbeitern, Angestellten, Handwerkern, Selbständigen und Landwirten die männliche Mortalität doppelt so stark ist wie bei der weiblichen.

Wanner (2012) zufolge ist jedoch vor allem klar, dass das Bildungsniveau ein signifikanter Prädiktor der Lebenserwartung ist. So zeigt sich, dass im Jahr 2000 für männliche 25-Jährige ohne Ausbildung eine Lebenserwartung von 50.8 Jahren, mit Tertiärabschluss dagegen 55.7 Jahren geschätzt wurde. Bei den Frauen betrug die Differenz 57.2 zu 60.2 Jahre. Bei den 65Jährigen sind die Differenzen etwas geringer: 16 zu 18.7 Jahre bei den Männern, 20.4 zu 22.4 Jahren bei den Frauen.

Dieser Befund stimmt mit US-amerikanischen Befunden (Chetty et al. 2014; Sasson 2016) überein, wonach die geschlechtsspezifischen Unterschiede mit zunehmendem Status abnehmen. Mit zunehmender Bildung und Einkommen verringert sich demnach der Lebenszeitvorteil der Frauen.

Dass sich der Unterschied zwischen Männern und Frauen zumindest in der westlichen Welt mit zunehmendem sozialem Status verringert (Abbildung 1), wird auch von den oben erwähnten Studien aus Deutschland belegt. Eine mögliche Erklärung dafür verweist auf den Befund, dass sich weniger qualifizierte Frauen eher für ein traditionelles Familienmodell als für einen riskanten Männerberuf wie etwa die Bauarbeit entscheiden. Nimmt man zudem an, dass die lebensbedrohlichen Risiken der bezahlten und unbezahlten Arbeit bei den Frauen weniger streuen als bei den Männern, liegt es auf der Hand, dass die Lebenserwartungskluft mit zunehmendem Status konvergiert.

\section{Abbildung 1: Lebenserwartungskluft zwischen Männern und Frauen}

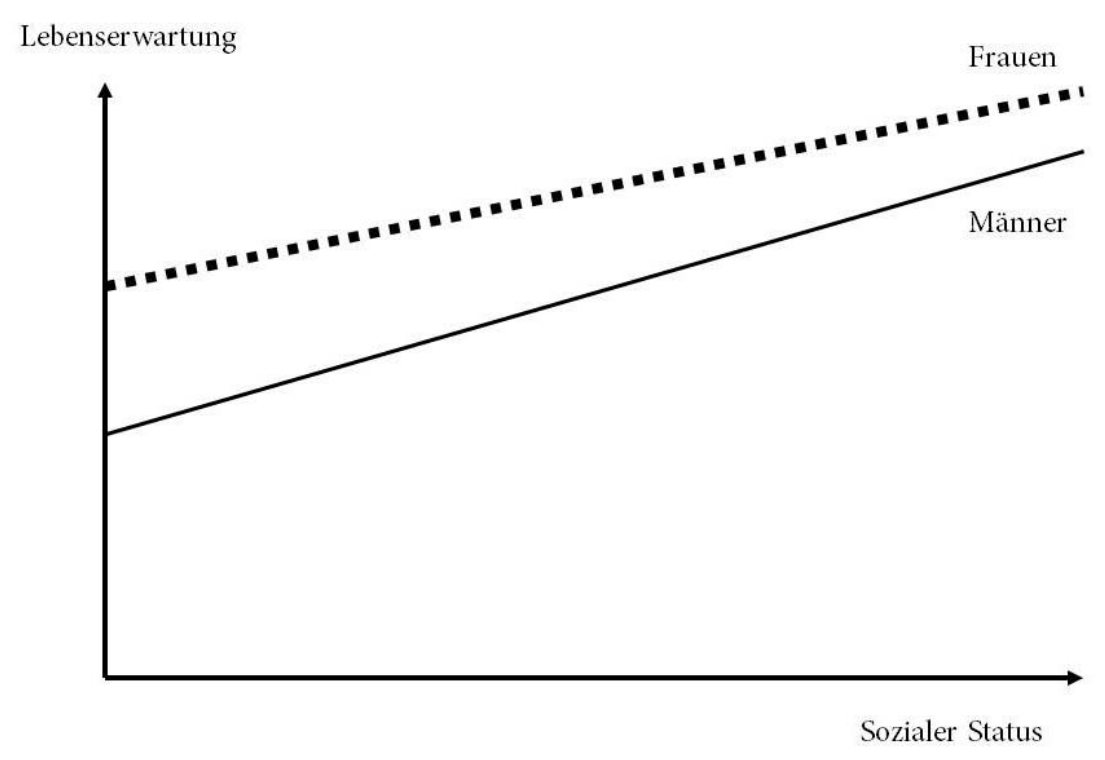

Quelle: Eigene Darstellung 
Besonders aufschlussreich in Bezug auf die Schweiz sind vor allem auch die Analysen von Moser et al. (2014). Obwohl ihre Analysen vornehmlich regionale Mortalitätsunterschiede beleuchten, ist auch ein multivariater Test verschiedener Faktoren enthalten, die die Mortalität in sogenannten Nachbarschaften (1.3 Millionen sich überlappender Cluster von 50 Haushalten) beeinflussen. So zeigt sich in Bezug auf die Lebenserwartung in den Nachbarschaften in der Schweiz, dass die Lebenserwartung mit zunehmendem Einkommen und Bildung ansteigt. Lebensverlängernd wirken auch die Heirat im Vergleich zum Single-, Wittwer- oder Geschiedenendasein. Bemerkenswert ist vor allem auch, dass SchweizerInnen eher geringere Lebenserwartungen aufweisen als AusländerInnen. Bei den Berufen zeigt sich, dass Management und zertifizierte Professionen eher lebensverlängernd, mangelnde Qualifikationen und vor allem Arbeitslosigkeit dagegen eher lebensverkürzend wirken.

Vergleichsweise selten wird der Zusammenhang zwischen Kontextvariablen und der durchschnittlichen Lebenserwartung überprüft. Bemerkenswert ist indes, dass das Bruttoinlandprodukt pro Kopf nicht linear mit der durchschnittlichen Lebenserwartung korreliert. So steigt die Lebenserwartung mit zunehmendem wirtschaftlichem Entwicklungsstand in den armen Ländern stark an. Bei den reichen Ländern bringen Entwicklungsvorsprünge dagegen immer geringere Vorsprünge bei der Lebenserwartung. Für Wilkinson und Pickett (2009) ist das Verschwinden des Zusammenhangs in den wirtschaftlich starken Ländern ein Indiz dafür, dass in den reichen Ländern vorab die sozioökonomische Ungleichheit, gemessen am GiniIndex, die durchschnittliche Lebenserwartung beeinflusst. So belegen zumindest bivariate Korrelationsanalysen für OECD-Länder und für US-Bundesstaaten, dass mit zunehmender Ungleichheit einerseits die Kindersterblichkeit zunimmt und andererseits die durchschnittliche Lebenserwartung sinkt.

Noch mangelt es vor allem an Studien, die gleichermassen Effekte von individuellen Merkmalen und Kontextmerkmalen auf die Lebenserwartung überprüfen. Wenn wir davon ausgehen, dass mit einer geringeren Gesundheit ein höheres Mortalitätsrisiko einhergeht, können wir Chuang et al. (2013) zufolge auf der Basis von Daten zur Selbsteinschätzung der Gesundheit in 29 Ländern immerhin vermuten, dass neben dem Geschlecht, der Bildung und dem Einkommen auch das Ausmass der sozialen Inklusion, des Sozialkapitals und der sozialen Diversität die Lebenserwartung beeinflussen. So zeigt sich, dass u.a. Gesundheitsausgaben, ethnische Toleranz, Bildungsausgaben, generalisiertes Vertrauen, demokratische Werte, Vereinsmitgliedschaften, Immigration und freiheitliche Werte gesundheitsfördernd wirken.

Über alle regionalen Grenzen hinweg spricht die empirische Forschung jedenfalls für persistente geschlechts- und schichtspezifische Unterschiede in der Mortalität und Lebenserwartung. Auch wenn nicht zu negieren ist, dass sich diese Ungleichheiten, gemessen etwa an der Säuglingssterblichkeit, in den letzten Jahrhunderten in der OECD-Welt dank einer modernen wohlfahrtsstaatlichen abgesicherten Gesundheitsvorsorge verringert haben, ist absehbar, dass eine Neoliberalisierung der Sozialpolitik die Kluft zwischen den Lebenszeitchancen wieder vertieft, und zwar vor allem deshalb, weil sowohl die ökonomischen und kulturellen Ungleichheiten als auch die Versorgungsungleichheiten („Zweiklassenmedizin“, siehe Kalvelage 2014) wieder ansteigen (vgl. auch Coburn 2014).

Welches Lebensalter jemand erreicht, wird folglich auch in naher und mittlerer Zukunft nicht nur vom Zufall eines Unfalls, eines Gewaltverbrechens oder genetischen Vor- und 
Nachteilen, sondern vor allem auch vom sozialen Herkunftsmilieu und den damit ererbtem ökonomischem, kulturellem und sozialen Kapital und dem erreichten sozialen Status beeinflusst. Die Lebenszeitchancen von Menschen mit ungünstigen Herkunft- und Statuskombinationen sind demnach soweit eingeschränkt, dass wir nach wie vor schlussfolgern können, dass Arme früher sterben als Reiche.

\section{Gesundheitliche Chancenungleichheiten}

Zahlreiche soziologische Analysen belegen, dass selbst die wohlhabenden OECD-Länder trotz Chancengleichheitsrhetorik immer noch weit davon entfernt sind, das meritokratische Ideal der Leistungsgesellschaft zu realisieren. So verweisen statistisch abgesicherte Statuszuweisungsmodelle darauf hin, dass der soziale Status von Menschen noch immer von der sozialen Herkunft und weiteren askriptiven Merkmalen wie Geschlecht, Hautfarbe, aber auch Herkunfts- und Wohnort beeinflusst werden, und zwar sowohl direkt als auch indirekt über schulischen Erfolg oder Sozialkapital.

Gesundheitssoziologische Analysen belegen zudem enge Zusammenhänge zwischen sozialem Status und der Lebenserwartung, wobei in der Regel drei Faktorenbündel als Gründe für das erhöhte Mortalitätsrisiko statustiefer Gruppen thematisiert werden (z.B. Janssen 2011). Vorab wird argumentiert, dass Menschen über unterschiedliche Ressourcen verfügen, um mit Gesundheitsrisiken und Krankheiten umzugehen. Wichtig sind allen voran Kenntnisse über gute Ernährung und gesundheitsfördernde und -hemmende Faktoren wie etwa Bewegungsmangel, Übergewicht, Rauchen und Alkoholkonsum. Es liegt auf der Hand, dass diese Kenntnisse mit zunehmendem Bildungsniveau stärker vorhanden sind. Positiv auf die Gesundheit wirken sich aber auch die Verfügbarkeit von finanziellen Ressourcen und sozialem Kapital aus. So bietet ökonomisches Kapital den Zugang zur Spitzenmedizin, und gute soziale Beziehungen sind vorteilhaft bei der Arztwahl und der Bewältigung von Krankheiten. Wichtig sind aber auch Belastungen am Arbeits- und Wohnort. So ist absehbar, dass gesundheitliche Belastungen, seien es die Unfallgefahr und toxische Risiken am Arbeitsplatz oder die Schadstoffoder Lärmbelastung am Wohnort, bei Menschen mit wenig Einkommen virulenter ist. Entscheidend sind schliesslich auch Unterschiede bei der medizinischen Versorgung. So ist nicht zu übersehen, dass ärmere Menschen zwar häufig nicht bei der Grundversorgung, aber u.U. bei der teuren Spitzenmedizin benachteiligt sind. Menschen mit wenig Bildung und Einkommen sind also sowohl bezüglich gesundheitlich relevanter Ressourcen als auch bezüglich Belastungen und medizinischer Versorgung beeinträchtigt und verfügen folglich über geringere Lebenszeitchancen.

Statusunterschiede lassen sich demnach vorab darin erkennen, ob ein Mensch raucht, trinkt, sich bewegt und übergewichtig ist. Sehr gut belegt ist der Zusammenhang zwischen Status und Rauchkonsum. So sprechen die meisten Studien dafür, dass die Intensität des Rauchens mit zunehmendem Status abnimmt. Das trifft auch für die Schweiz zu (Boes et al. 2016). Mit zunehmendem sozialen Status nimmt auch die Bereitschaft zu freiwilligen körperlichen Aktivitäten zu (Beenackers et al. 2012). Auch dieser Zusammenhang lässt sich in der Schweiz nachweisen (Boes et al. 2016), genauso wie eine Verringerung von Übergewicht mit zunehmendem Status. Dagegen lassen sich beim Alkoholkonsum länderspezifische Unterschiede 
erkennen. Während Elgar et al. (2005) und Probst et al. (2014) Alkohol als signifikanten Faktor für die Erklärung des erhöhten Mortalitätsrisikos statustiefer Schichten sehen, belegen die Befunde von Boes et al. (2016) für die Schweiz einen inversen Zusammenhang. Demnach steigt der Alkoholkonsum in der Schweiz mit zunehmendem Status.

Die Befunde von Boes et al. (2016) sind für uns von besonderem Interesse, da sie auf der Schweizerischen Gesundheitsbefragung, einer repräsentativen Stichprobe der ständigen Wohnbevölkerung in der Schweiz in den Jahren 1997, 2002, 2007 und 2012 beruhen. Der Vergleich der vier Stichproben erlaubt auch, Trends zu erkennen. So fällt beim Alkoholkonsum nicht nur der inverse Zusammenhang auf. Es zeigt sich auch, dass sich der riskante Alkoholkonsum verringert hat, vor allem bei den reichsten 20\%. Auch der Tabakkonsum ist rückläufig, wobei noch 1997 die zwei obersten Einkommensquintile am meisten regelmässige RaucherInnen aufwiesen. Dagegen lässt sich bei den körperlichen Aktivitäten zwischen 1997 und 2012 keine Veränderung des Zusammenhangs erkennen. So nimmt der Aktivitätsgrad in allen Quintilen zu. Problematisch ist hingegen der Trend beim Ernährungsbewusstsein. So zeigt sich, dass das oberste Einkommensquintil mehr, das unterste hingegen weniger auf die Ernährung achtet. Zudem zeigt sich bei der Fettleibigkeit, dass sich zwar nicht die Ungleichheit zwischen den Quintilen, aber die Prävalenz markant erhöht hat.

Dass Gesundheit etwas mit Statusunterschieden und damit letztlich auch mit Chancenungleichheiten zu tun hat, ist auch den politisch verantwortlichen Behörden bewusst. So betont etwa die Weltgesundheitsorganisation den Einfluss sozialer Faktoren auf die Gesundheit: „we must address the conditions in which people are born, grow, live, work and age, which are the key determinants of health equity“" (WHO 2014, xviii).

Auch für das schweizerische Bundesamt für Gesundheit (BAG) ist neben der Lebens- und Versorgungsqualität sowie der Transparenz die Chancengleichheit ein zentrales Ziel des Gesundheitswesens. Ausgehend von Artikel 2, Absatz 3 der Bundesverfassung - „Sie (die Eidgenossenschaft, $\mathrm{MN}$ ) sorgt für möglichst grosse Chancengleichheit unter den Bürgerinnen und Bürgern" - räumt das Factsheet zum Handlungsfeld Chancengleichheit (BAG 2014) zunächst explizit ein:

„Obwohl die Medizin in den letzten Jahren beachtliche Fortschritte erzielt hat, gibt es Hinweise, dass auch in der Schweiz nicht alle die gleichen Chancen für eine bestmögliche Gesundheit haben: Personen mit niedriger Bildung, tieferer beruflicher Stellung oder geringem Einkommen sterben deutlich früher als die übrige Bevölkerung. Zudem leiden sie in ihrem kürzeren Leben häufiger an gesundheitlichen Beeinträchtigungen. Sind ganze Bevölkerungsgruppen betroffen (z.B. armutsgefährdete / armutsbetroffene Kinder und Jugendliche), hat sich dafür international der Begriff der "gesundheitlichen Ungleichheit» durchgesetzt."

In der Folge wird eine Liste von Massnahmen präsentiert, die mithelfen, mehr gesundheitliche Chancengleichheit zu realisieren:

- periodisches Monitoring der Verteilung der Krankheitsrisiken und der Gesundheitschancen in der Bevölkerung als Grundlage für Massnahmen;

- Sicherstellung einer nachhaltig solidarisch abgestützten Finanzierung der Krankheitskosten;

- ein chancengleicher Zugang zur Prävention, Kuration, Pflege und Betreuung; 
- strukturelle Massnahmen ausserhalb des Gesundheitswesens, welche die Gesundheit fördern, da gut belegt ist, dass gesundheitsschädliches Verhalten wesentlich von den Lebensbedingungen mitbestimmt wird.

Vor dem Hintergrund der drei oben erwähnten empirisch belegten Mechanismen (Ressourcen, Belastung, Vorsorge) fällt auf, dass nicht nur die medizinische Versorgung, sondern auch die Belastungen (Lebensbedingungen) als Adressat von Massnahmen gesehen werden. Das wird auch daran erkennbar, dass zwischen einem Ansatz beim Gesundheitssystem, einem Ansatz beim Lebensstil und den gesundheitlichen Belastungen unterschieden wird. Ausgeklammert, weil nicht beeinflussbar, bleibt einzig die Verfügbarkeit von ökonomischem, kulturellem und sozialem Kapital.

\section{Implikationen für die Altersvorsorge}

Die Forderung nach Chancengleichheit ist nicht nur in der Schweiz ein zentrales Anliegen moderner Sozialpolitik. Entsprechend fordert die moderne Bildungspolitik, dass schulische Leistungen nicht mehr vornehmlich von der sozialen Herkunft und anderen askriptiven Merkmalen abhängen. Analog steht neuerdings auch in der Gesundheitspolitik die Forderung im Vordergrund, dass der soziale Status nicht mehr die Gesundheits- und Unfallrisiken und die medizinische Versorgungsqualität bestimmten soll.

Nicht zuletzt soziologische Analysen zeigen jedoch, dass die dazu entwickelten Massnahmen nur graduell greifen, und zwar vor allem auch deshalb, weil es Eltern nach wie vor erlaubt ist, ihr soziales, ökonomisches und kulturelles Kapital an ihre Kinder zu vererben. Diese nicht zuletzt in schichtspezifischen Lebenserwartungen erkennbaren Handicaps lassen sich durch Aufklärungsarbeit in der Schule, arbeitsrechtliche Massnahmen und eine ökologisch sensible Wohnungspolitik allenfalls verringern, aber nicht beseitigen.

Wohlwissend, dass sich die gesundheitlichen Ungleichheiten und damit ungleichen Lebenszeitchancen ohne einen radikalen Strukturwandel nicht beseitigen lassen, könnte man folglich auf die Frage fokussieren, ob es nicht ein Gebot der Fairness wäre, in der Altersvorsorge jene sozialen Gruppen zu begünstigen, die aufgrund struktureller Benachteiligungen über geringere Lebenszeitchancen verfügen.

Fakt ist indes zum einen, dass in der Altersvorsorge seit einigen Jahren ausschliesslich die Finanzierungsfrage im Vordergrund steht. So sei absehbar, dass sich mit der zunehmenden Lebenserwartung der Bevölkerung die Relation zwischen jenen, die Beiträge bezahlen, und jenen, die Renten beziehen, zuungunsten der Jungen verschlechtere und damit einerseits den sogenannten Generationsvertrag und andererseits die Finanzierung der Altersvorsorge bedrohe.

Zum andern ist nicht zu übersehen, dass kaum jemand die Streuung der Lebenserwartung thematisiert. In der Regel wird unterstellt, dass ein Rentner mit 83 Jahren und eine Rentnerin mit 85 Jahren stirbt, und das, obwohl bereits ein Blick in die Todesanzeigen von Tageszeitungen genügt, um diese Prämisse zu hinterfragen.

Mit anderen Worten: Es wird ignoriert, dass die Kombination von ungleichen Lebenschancen, konstanter Beitragsdauer und fixem Rentenalter impliziert, dass Menschen je nach sozialen Status unterschiedlich lange Vorsorgegelder beziehen. Wer im Gastgewerbe arbeitet 
und Beitragslücken aufweist, wird selbst nach der Einführung des Rauchverbots in Restaurants folglich im ungünstigsten Fall immer noch dreifach bestraft: durch ein geringes Einkommen, eine gekürzte Rente und ein kürzeres Leben.

Die Forderung, dass bestimmte Gruppen von Lohnarbeitenden über ein erhöhtes Mortalitätsrisiko verfügen und folglich in den Genuss einer vorzeitigen Pensionierung kommen sollten, ist an sich nicht unbekannt. Gerechtfertigt werden solche realisierte Modelle in Europa in der Regel mit dem Hinweis auf die eingeschränkte Lebenserwartung gewisser Beschäftigten:

"The rationale for this scheme[s] is that hazardous or arduous work increases mortality and reduces life expectancy, thus reducing the time during which retirement benefits can be enjoyed. This results in such workers being made eligible for earlier access to pension benefits than otherwise available in country's general pension scheme." (Zaidi/Whitehouse 2009: 4)

Im Vordergrund standen bislang besonders stark belastete Tätigkeiten. Prominente Beispiele sind Bergarbeiter wie etwa bei der deutschen "knappschaftlichen Rentenversicherung" oder Schwerarbeiter wie etwa bei der österreichischen Schwerarbeitpension (vgl. Zaidi/Whithouse 2009; Brussig et al. 2011). Analog dazu finden wir inzwischen selbst in der liberalen Schweiz einen Gesamtarbeitsvertrag, der es BauarbeiterInnen eine Rente ab 60 Jahren ermöglicht. Anlass dafür war nicht zuletzt die erwähnte Studie von Guberan und Usel (2000).

Problematisch bei der Begünstigung von Berufsgruppen ist allerdings vorab, dass nicht alle benachteiligten Berufsgruppen in den Genuss solcher Regelungen kommen. In der Tat sind nicht Sterbetafeln ausschlaggebend, sondern die politische Macht organisations- und konfliktfähiger Gewerkschaften. Entsprechend geniessen gewisse Berufsgruppen wie etwa Beamte oder Flugpiloten auch ohne unterdurchschnittliche Lebenserwartung eine Frühverrentung. Hinzu kommt, dass die berufliche Vorsorge nur die erwerbstätigen Beschäftigten erfasst, also u.a. die älteren Arbeitslosen ohnehin ausschliesst.

Auch wenn der Belastungsgrad der individuellen Arbeit ein objektiveres Kriterium für eine vorzeitige Pensionierung wäre (Brussig et al. 2011), bliebe damit wiederum die Problematik der intragenerationellen Mobilität und der Wechsel zwischen wenig und stark belasteten Tätigkeiten ungelöst. Zudem ist - wie der Überblick über die Forschung zeigt - die Belastung in der Arbeitswelt nur ein Faktor u.a., der die Mortalität und Lebenserwartung beeinflusst.

Als Kriterium für eine ungleichheitssensible Flexibilisierung des Rentenalters würden sich zweifellos vorab das Einkommen und die Bildung anbieten. Zumal sich das Einkommen im Unterschied zur Bildung während des Lebenszyklus vergleichsweise stärker verändert und schwierig messbar ist, spricht allerdings einiges für die Berücksichtigung der Ausbildung oder die Anzahl der Schuljahre, die ein Mensch absolviert hat. Kurzum: Menschen mit wenig Qualifikationen und folglich limitierter Lebenserwartung dürften demnach früher in den Ruhestand als AkademikerInnen (vgl. auch Wanner 2012).

Auch wenn die ungleichen Lebenszeitchancen von Menschen empirisch nicht zu negieren sind und nicht zuletzt die frühere Verrentung der Frauen trotz höherer Lebenserwartung mit strukturellen Benachteiligungen gerechtfertigt wurde, dürfte eine ungleichheitssensible Flexibilisierung des Rentenalters in der liberalen Schweiz zweifellos nur wenig Resonanz findet. So wird im hegemonialen, von neoliberalem Denken geprägten Eigenverantwortungsdiskurs die Ansicht die Oberhand behalten, dass für eine tiefe oder hohe Lebenserwartung und damit für 
die Dauer des von der Altersvorsorge finanzierten Lebensabends ausschliesslich die Beschäftigten selbst verantwortlich sind.

\section{Schlussfolgerungen}

Der Tod ist kein Zufall. Dass Menschen tödlich verunfallen oder Opfer von Naturkatastrophen werden, wird auf den ersten Blick häufig als Ergebnis von Zufällen, Schicksal oder Wille Gottes interpretiert. Auf den zweiten Blick wird allerdings kaum jemand negieren, dass sich die durchschnittliche Lebenszeit dank der medizinischen und sozialpolitischen Modernisierung in den letzten beiden Jahrhunderten massiv erhöht hat.

Dank der Instrumente der empirischen Sozialforschung wissen wir aber auch, dass die Lebenszeitchancen von Menschen ungleich verteilt sind und eine Vielzahl sozialer Faktoren die Lebenserwartung und Mortalität und damit letztlich die Lebenszeitchancen zwar nicht determinieren, aber doch mehr oder weniger stark beeinflussen. Männer, die wenig gebildet sind, sich ungesund ernähren, Drogen konsumieren, gefährliche Jobs ausüben und/oder in Gegenden wohnen, die stark von Schadstoffen belastet sind, leben in der Regel weniger lang als Akademikerinnen, die sich ein gesundes Leben leisten können. Kurzum: Nicht die betroffenen Menschen, sondern die gesellschaftlichen Rahmenbedingungen sind letztlich dafür verantwortlich, dass die Gefahr, Opfer eines Gewaltverbrechens oder einer tödlichen Krankheit zu werden, in lateinamerikanischen Favelas grösser ist als auf Island.

Auch wenn inzwischen moderne Wohlfahrtsstaaten tieferen sozialen Schichten den Zugang zu einer guten medizinischen Versorgung ermöglichen, bleiben die Lebenszeitchancen dennoch ungleich verteilt. In vielen Ländern wie etwa der Schweiz sind sich die Gesundheitsbehörden dieser Ungleichheiten durchaus bewusst und versuchen, sie durch präventive Massnahmen zu verringern. So können wir, auch wenn wir über keine Längsschnittanalysen verfügen, zumindest unterstellen, dass ungleichheitssensible Gesundheitspolitiken nicht nur zum Anstieg der durchschnittlichen Lebenserwartung, sondern auch zu einer Verringerung der statusbedingten Lebenszeitungleichheiten beigetragen haben.

Allein diese beiden Erfolge vermögen jedoch nicht darüber hinweg täuschen, dass in ärmeren Ländern nach wie vor tiefe Lebenserwartungen und starke Statuseffekte zu beobachten sind (z.B. Human Development Report 2005). Zudem beunruhigt, dass inzwischen einige Studien dafür sprechen, dass der Konvergenztrend bezüglich der schichtspezifischen Lebenserwartung spätestens seit Ende des 20. Jahrhunderts gestoppt scheint (Abbildung 2) und sich seither die Unterschiede zwischen den Schichten vor allem auch in den reicheren Ländern wieder akzentuieren. Mit anderen Worten: Der gut dokumentierte Anstieg der sozioökonomischen Ungleichheiten in der westlichen Welt scheint sich u.a. in grösseren Lebenserwartungsund Mortalitätsungleichheiten zwischen den sozialen Schichten niederzuschlagen. Falls dieser Trend anhält, liegt es auf der Hand, dass auch die Lebenszeitungleichheiten der RentnerInnen wieder ansteigen. 
Abbildung 2: Trends der Lebenserwartungskluft zwischen sozialen Schichten

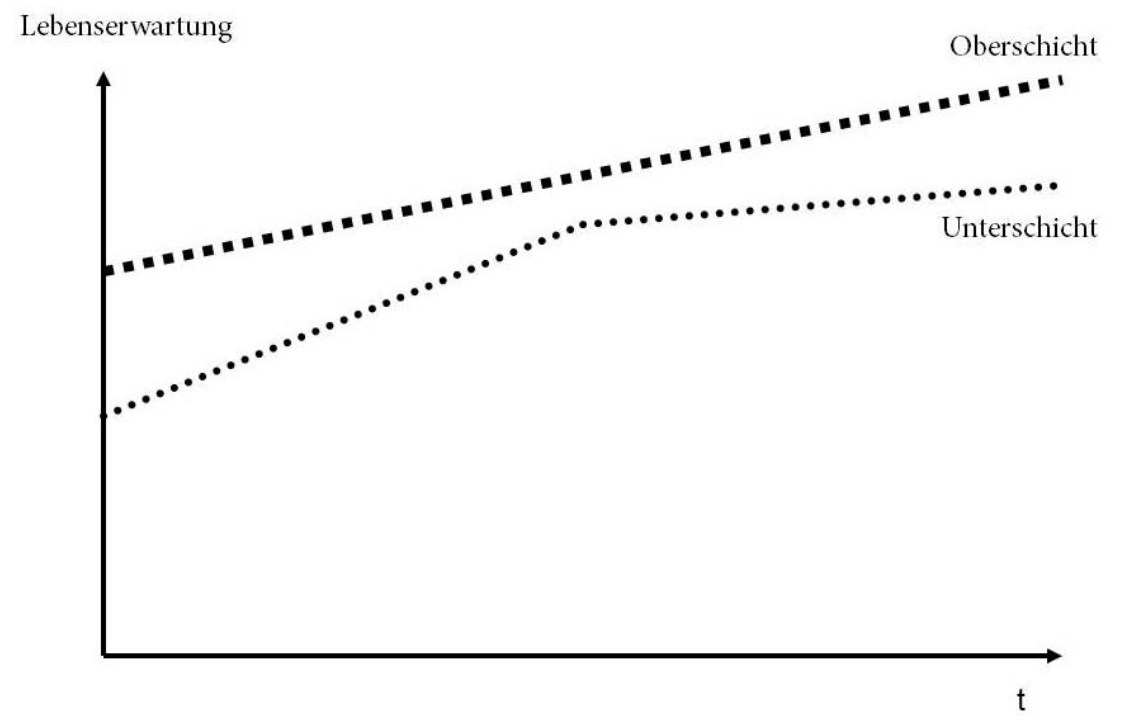

Quelle: Eigene Darstellung

Trotz empirischer Evidenz ignorieren die meisten Systeme der Altersvorsorge, dass die Lebenszeitchancen ungleich verteilt sind. So negiert auch der schweizerische Diskurs über die Neujustierung des Rentenalters, dass für die Lebensdauer von Menschen auch sozialstrukturelle Faktoren verantwortlich sind. Aussen vor bleibt damit, dass diesbezüglich benachteiligte Menschen nicht nur im Arbeitsleben benachteiligt sind, sondern auch eine geringere Rente weniger lang beziehen.

Ausnahmen von dieser Regel bilden bislang einzig ausgewählte Beschäftigte, deren Beruf und/oder Tätigkeit mit besonderen gesundheitlichen Belastungen verbunden sind. In vielen Ländern können daher Mitglieder gewisser Berufsgruppen vorzeitig in den Ruhestand. In der Schweiz profitieren vorab die Bauarbeiter davon, erlaubt ihnen der Gesamtarbeitsvertrag doch, sich bereits mit 60 Jahren aus dem Berufsleben zurückzuziehen.

Allerdings bleibt auf den ersten Blick unklar, weshalb nicht auch andere besonders belastete Beschäftigte früher in die Rente gehen können. Betrachtet man die Sonderregelungen in anderen Ländern, wird auf den zweiten Blick allerdings deutlich, dass die meisten Regelungen vornehmlich professionspolitischem Engagement zu verdanken sind. Entsprechend ist nicht zu vermeiden, dass auch Erwerbstätige in den Genuss des Vorruhestands kommen, die bezüglich Lebenserwartung nicht benachteiligt sind. Aus sozialpolitischer Sicht ist es daher äusserst fragwürdig, wenn nicht vornehmlich ältere Arbeitslose oder besonders belastete, sondern organisations- und konfliktfähige Gruppen über die Option einer Frühverrentung verfügen.

Ausgehend von empirischen Befunden würde sich vor allem das Bildungsniveau als leicht handbares Kriterium für den Zeitpunkt der Pensionierung anbieten. In der Tat zeigen alle Studien, dass mit zunehmender Anzahl an Bildungsjahren die Lebenserwartung ansteigt. Von daher liessen sich bildungsbedingte Ungleichheiten vergleichsweise leicht durch unterschiedliche Renteneintrittsalter kompensieren. Allerdings ist leicht absehbar, dass dieses Anliegen momentan keine Realisierungschance hat. So werden im hegemonialen Altersvorsorgediskurs, wonach für das eigene Leben weder Gott noch die gesellschaftlichen Rahmenbedingun- 
gen, sondern vornehmlich jeder Mensch selbst verantwortlich ist, auch in Zukunft die ungleichen Lebenszeitchancen und damit die Unfairness des fixen Rentenalters ignoriert.

\section{Literaturverzeichnis}

BAG (2014). Factsheet Handlungsfeld Chancengleichheit. Bern: Bundesamt für Gesundheit.

Beenackers, Marielle A., Carlijn B.M. Kamphuis, Katrina Giskes, Johannes Brug, Anton E. Kunst, Alex Burdorf and Frank J. van Lenthe (2012). Socioeconomic inequalities in occupational, leisure-time, and transport related physical activity among European adults: A systematic review. International Journal of Behavioral Nutrition and Physical Activity 9, 116.

Boes, Stefan, Cornel Kaufmann und Joachim Marti (2016). Sozioökonomische Ungleichheiten im Gesundheitsverhalten der Schweizer Bevölkerung. Obsan Dossier 51.

Brussig, Martin Andreas Jansen und Matthias Knuth (2011). Differenzierte Altersgrenzen in der Rentenversicherung aufgrund beruflicher Belastungen? Vorüberlegungen für ein empirisches Konzept. WISO Diskurs, Dezember 2011.

Chetty, Raj, Michael Stepner, Sarah Abraham, Shelby Lin, Benjamin Scuderi, Nicholas Turner, Augustin Bergeron und David Cutler (2016). The association between income and life expectancy in the United States, 2001-2014.

Chuang, Ying-Chih, Kun-Yang Chuang, Tzu-Hsuan Yang (2013). Social cohesion matters in health. International Journal for Equity in Health, 12-87.

Coburn, David (2014). Neoliberalism and Health. The Wiley Blackwell Encyclopedia of Health, Illness, Behavior, and Society, 1678-1683.

Cordazzo, Valérie (2006). Die Sterblichkeit der Schweizer Geburtsjahrgänge 1900 bis 2030. Neuenburg: demos - Informationen aus der Demografie, 3/2006.

Eriksson, Malin (2011. Social capital and health implications for health promotion. Global Health Action 4(5611), 1-11.

Elgar, Frank J., Chris Roberts, Nina Parry-Langdon, and William Boyce (2005). Income inequality and alcohol use: a multilevel analysis of drinking and drunkenness in adolescents in 34 countries. European Journal of Public Health 15(3), 245-250.

Gubéran, Etienne und Massimo Usel (2000). Mortalité prématurée et invalidité selon la profession et la classe sociale à Genève. Genf: OCIRT.

Hartung, Susanne (2014). Sozialkapital und gesundheitliche Ungleichheit. Wiesbaden: Springer.

Human Development Report (2005). International cooperation at a crossroads. Aid, trade and security in an unequal world. New York: UNDP.

Janssen, Christian (2011). Ein medizin-soziologischer Überblick über den Zusammenhang zwischen sozialer Ungleichheit und Gesundheit. Bayerische Sozialnachrichten 1/2011, 3-9.

Kalvelage, Bernd (2014). Klassenmedizin. Plädoyer für eine soziale Reformation der Heilkunst. Berlin: Springer.

Künzler, Gabriela und Carlo Knöpfel (2002). Arme sterben früher. Soziale Schicht, Mortalität und Rentenalterspolitik in der Schweiz. Luzern: Caritas-Verlag. 
Lampert, Thomas und Lars Eric Kroll (2014). Soziale Unterschiede in der Mortalität und Lebenserwartung. GBE kompakt 5(2).

Mackenbach, Johan P., Irina Stirbu, Albert-Jan R. Roskam, Maartje M. Schaap, Gwenn Menvielle, Mall Leinsalu und Anton E. Kunst (2008). Socioeconomic inequalities in health in 22 countries. New England Journal of Medicine, 358, 2468-2481.

Mackenbach, Johan P., Ivana Kulhánová, Barbara Artnik, Matthias Bopp, Carme Borrell, Tom Clemens, Giuseppe Costa, Chris Dibben, Ramune Kalediene, Olle Lundberg, Pekka Martikainen, Gwenn Menvielle, Olof Östergren, Remigijus Prochorskas, Maica Rodríguez-Sanz, Bjørn Heine Strand, Caspar W.N. Looman und Rianne de Gelder (2016). Changes in mortality inequalites over two decades: register based study of European countries. BMJ 353, i1732, 1-8.

Moser, André, Radoslaw Panczak, Marcel Zwahlen, Kerri M. Clough-Gorr, Adrian Spoerri, Andreas E. Stuck und Matthias Egger (2014). What does your neighbourhood say about you? A study of life expectancy in 1.3 million Swiss neighbourhoods. J Epidemiol Community Health 68, 1125-1132.

Perrenoud, Alfred (1975). L'inégalité sociale devant la mort à Genève au XVII siècle. Population 30, 221-243.

Poder, Thomas und Jie He (2010). Social capital and health: What the empirical literature teaches us? Global Journal of Health Science 2(1), 3-7.

Probst, Charlotte, Michael Roerecke, Silke Behrendt und Jürgen Rehm (2014). Socioeconomic differences in alcohol-attributable mortality compared with all-cause mortality: a systematic review and meta-analysis. International Journal of Epidemiology 43(4), 1314-1327.

Rocco, Lorenzo und Marc Suhrcke (2012). Is Social Capital good for Health? A European Perspective. Copenhagen: WHO Regional Office for Europe.

Sasson, Isaac (2016). Trends in life expectancy and lifespan variation by educational attainment: United States, 1990-2010. Demography 53, 269-293.

Therborn, Göran (2013). The Killing Fields of Inequality. Cambridge: Polity Press.

Voges, Wolfgang und Olaf Groh-Samberg (2011). Der Einfluss von Einkommenslage und Lebenslage auf das Mortalitätsrisiko. ZeS Report 16(1), 1-7.

Wanner, Philippe (2012). Unterschiedliche Sterblichkeit: Auswirkungen auf die Sozialversicherungen. Soziale Sicherheit CHSS 6, 351-356.

Wilkinson, Richard und Kate Pickett (2009). The Spirit Level. Why More Equal Societies Almost Always Do Better. London: Allen Lane.

WHO (2014). Review of Social Determinants and the Health Divide in the WHO European Region. Copenhagen: World Health Organization.

Zaidi, Asghar und Edward Whitehouse (2009). Should Pension Systems Recognise "Hazardous and Arduous Work?" OECD Social Employment and Migration Working Papers, No. 91, OECD Publishing. 